\title{
Flotillin-1 is essential for PKC-triggered endocytosis and membrane microdomain localization of DAT
}

\author{
M. Laura Cremona ${ }^{1, \ddagger}$, Heinrich J.G. Matthies ${ }^{2}$, Kelvin Pau $^{1}$, Erica Bowton ${ }^{2}$, Nicole Speed ${ }^{2}$, \\ Brandon J. Lute ${ }^{2}$, Monique Anderson ${ }^{1}$, Namita Sen ${ }^{3}$, Sabrina D. Robertson ${ }^{2}$, Roxanne A. \\ Vaughan $^{4}$, James E. Rothman ${ }^{5}$, Aurelio Galli ${ }^{2}$, Jonathan A. Javitch ${ }^{3}$, and Ai Yamamoto ${ }^{1, *}$ \\ ${ }^{1}$ Departments of Neurology, and Pathology and Cell Biology, Columbia University, College of \\ Physicians and Surgeons, New York, NY 10032 \\ 2Department of Molecular Physiology and Biophysics, Center for Molecular Neuroscience, \\ Kennedy Center, Vanderbilt University, Nashville, TN 37232 \\ ${ }^{3}$ Center for Molecular Recognition and Departments of Psychiatry and Pharmacology, Columbia \\ University, College of Physicians and Surgeons, New York, NY 10032 \\ ${ }^{4}$ University of North Dakota School of Medicine and Health Science, Grand Forks, ND \\ ${ }^{5}$ Department of Cell Biology, Yale University School of Medicine, New Haven, CT
}

\begin{abstract}
Plasmalemmal neurotransmitter transporters (NTTs) regulate the level of neurotransmitters, such as dopamine (DA) and glutamate, following their release at brain synapses. Stimuli including protein kinase $\mathrm{C}$ (PKC) activation can lead to the internalization of some NTTs and a reduction in neurotransmitter clearance capacity. We find that the protein Flotillin-1/Reggie-2 (Flot1) is required for PKC-regulated internalization of members of two different NTT families, the DA transporter (DAT) and the glial glutamate transporter EAAT2, and we have identified a conserved serine residue in Flot1 that is essential for transporter internalization. Further analysis revealed that Flot1 is also required to localize DAT within plasma membrane microdomains in stable cell lines, and is essential for amphetamine-induced reverse transport of DA in neurons but not for DA uptake. In sum, our findings provide evidence for a critical role of Flot1-enriched membrane microdomains in PKC-triggered DAT endocytosis and the actions of amphetamine.
\end{abstract}

\section{Introduction}

The NTTs are comprised of two structurally distinct families, the glutamate/neutral amino acid transporters (SLC1) and the neurotransmitter:sodium symporters (SLC6). Under steady-state conditions, some SLC1 gene family members such as the glial glutamate transporter (EAAT2), and some SLC6 members, such as the DAT, localize primarily to the

Users may view, print, copy, download and text and data- mine the content in such documents, for the purposes of academic research, subject always to the full Conditions of use: http://www.nature.com/authors/editorial_policies/license.html\#terms

*To whom correspondence should be addressed: Ai Yamamoto, Ph.D., 650 West $168^{\text {th }}$ Street, BB301, New York, NY 10032, ay46@columbia.edu.

\$New address: Department of Endocrinology, Columbia University, College of Physicians and Surgeons, New York, NY 10032 
plasma membrane. There, these transporters modulate the strength of neurotransmission by clearing neurotransmitters from the presynaptic space. Surface expression of DAT and EAAT2 can be modulated acutely by intracellular signaling events, such as PKC activation, which mobilize these NTTs into recycling endocytic compartments or to the lysosome. PKC-triggered internalization of DAT has been demonstrated predominantly in heterologous systems 1, 2, whereas internalization of EAAT2 has been shown in both heterologous and endogenous systems $3-6$.

Since the proposal of the 'lipid raft' hypothesis by Simons and Ikonen7, the biochemical and structural definition of these compartments in cellular membranes has been the subject of intense debate. Now termed 'membrane rafts', they are defined as small (10-200 nm), heterogeneous, highly dynamic, sterol- and sphingolipid-enriched domains that compartmentalize cellular processes8. Membrane rafts are commonly defined biochemically; their lipid composition leads to insolubility in cold, non-ionic detergents. Over the last several years, there has been growing evidence that DAT9-11 and EAAT212, 13 reside in membrane microdomains. Although membrane raft contributions to neurotransmitter transporter function have been proposed9-11, it has been technically difficult to ascertain the role of microdomain localization in transporter function. Interestingly, several proteins that reside in membrane rafts such as cholera toxin subunit B and glycosylphosphotidylinositol (GPi)-linked proteins, among others are internalized by non-clathrin mediated mechanisms14. Since lipids can scaffold key membrane-associated signaling events, such as activated PKC and src kinases, membrane raft localization may facilitate PKC-triggered internalization of DAT and EAAT2.

The best characterized mediators of clathrin-independent endocytosis are the caveolins such as caveolin-1 (Cav1). Cav1 can mediate rapid endocytosis15, 16 and bind to and be phosphorylated by PKC15, 16. Recently Flotillin-1/Reggie-2 (Flot1)17 was described as another potential mediator of endocytosis. Most frequently used as a marker for membrane rafts, Flot1 has been reported to delineate a discrete set of endocytic vesicles17 consistent with reports describing a rapid, caveolin-independent, but dynamin-dependent endocytic pathway involved in the formation of small, noncoated vesicles at the plasma membrane18, 19. Flot1 is highly conserved and widely expressed, especially in the nervous system $20,21$. A cargo specific for a Flot1-mediated endocytic pathway is still unknown. Although they share no sequence homology, interesting parallels can be drawn between Flot1 and Cav1 (Fig.S1). Both are membrane-associated proteins that orient similarly in the membrane in a cholesterol-dependent manner, multimerize through coiled-coil domains at their $\mathrm{COOH}$ termini and form hetero-oligomers leading to microdomains in the plasma membrane20, 22. Several phosphorylation sites have been identified in both, including sites for the Src kinase21, and importantly, for PKC21, 23.

Here we show that Flot1 is required for the PKC-triggered endocytosis of the NTTs DAT and EAAT2. Further analysis revealed that DAT forms a complex with palmitoylated Flot1 in a PKC-dependent manner, and that a conserved serine residue of Flot 1 is required for PKC-mediated internalization of DAT. Moreover, Flot1 is required to maintain DAT in membrane rafts, suggesting that membrane microdomain localization may be an important determinant of regulated endocytosis of DAT. Notably, despite previous results indicating 
that DAT localization influences its ability to transport DA; we found no detectable impact on DA uptake after Flot1 depletion. In contrast, depletion of Flot1 diminished amphetamineinduced reverse transport of DA in neurons. These findings indicate that membrane microdomain localization can influence both PKC-triggered internalization and amphetamine's actions on DAT.

\section{RESULTS}

Studies using heterologous systems have shown that DAT and EAAT2 rapidly internalize into endocytic structures in response to activation of PKC by the phorbol ester PMA1-6. As shown by both confocal microscopy and cell surface biotinylation, HEK293-derived EM4 cells stably expressing eYFP-tagged DAT (EM4-YFP-DAT)2 or HeLa cell lines stably expressing monomeric YFP24 (mYFP)-tagged DAT (HeLa-mYFP-DAT) or eGFP-tagged EAAT2 (HeLa-eGFP-ET2) demonstrate robust and rapid internalization of the transporter into early endosomal antigen 1 (EEA1)-positive vesicles in response to a 30 min exposure of PMA (Fig.1a-b, S1). It has previously been shown that the murine homolog of EAAT2, GLT-1, internalizes in response to PMA in neuronal-astrocyte co-cultures4, 6. To ascertain if this PKC-triggered event also is observed with endogenous DAT, we exposed primary dopaminergic neurons to $1 \mu \mathrm{M}$ PMA (direct PKC activation) for $30 \mathrm{~min}$ and immunostained for DAT. Confocal imaging revealed that PKC activation led to significant redistribution of endogenous DAT to an internal compartment in primary neurons (Fig.1c). Consistent with these findings, PMA treatment of mouse striatal slices led to a significant internalization of endogenous DAT, as shown by cell-surface biotinylation (Fig.1d). Thus, PKC-triggered internalization of DAT is evident not only in a heterologous system but also in primary neurons and brain slice preparations expressing endogenous DAT.

How is PKC-triggered internalization achieved? Mutagenesis of canonical PKC phosphorylation sites in DAT did not affect its PKC-regulated endocytosis in heterologous systems 25,26 , suggesting that rather than directly phosphorylating the transporter, PKC controls internalization by phosphorylating an unknown intermediary protein. Since structurally distinct NTTs can be internalized in response to PMA, and this PMA-triggered event is observed across a wide range of cells (Fig.1, S1), the intermediary protein may be a ubiquitous protein involved in fundamental cellular trafficking events. Pre-incubating EM4YFP-DAT with the reversible PKC inhibitor Gö6850 (also known as Bisindolmaleimide I or GF 109203X) inhibited PMA-triggered internalization in a dose-dependent manner (Fig.2ab). We hypothesized that overexpression of the requisite target of PKC for internalization would compete with the inhibitor for binding since Gö6850 reversibly binds the substrate binding site of PKC and consequently enhance internalization at low effective concentrations of the inhibitor (Fig.S2).

To determine the impact of membrane raft localization on PKC-triggered internalization of DAT, we first tested Cav1 (Fig. S3). Despite robust internalization in EM4-YFP-DAT cells (Fig.1), as previously reported for some HEK293 cells27, 28, we could not detect Cav1 protein in the HEK-293-derived EM4 cells (Fig.S3a)29. Furthermore, in the presence of Gö6850, Cav1 overexpression failed to blunt inhibition (Fig.S3b, c). In HeLa cells, which do express endogenous Cav1, small interfering RNA (siRNA)-mediated knockdown of Cav1 
also had no effect on PMA-triggered DAT internalization (Fig.S3d), further indicating that Cav1 is not required for this event.

We next turned to Flot1. Confocal imaging and cell surface biotinylation showed that overexpression of Flot1 in EM4-YFP-DAT diminished the Gö6850-mediated inhibition of PMA-triggered DAT internalization (Fig.2a-c): PMA-triggered endocytosis was assessed by confocal analysis as the number of cells that showed internalized DAT (into EAA1-positive compartments indicating entry into early endosomes) after exposure to PMA. Although 0.1 $\mu \mathrm{M}$ Gö6850 led to approximately $60 \%$ inhibition of PMA-triggered endocytosis, overexpression of Flot 1 significantly reduced this inhibition to $20 \%$. To ensure that overexpression of Flot1 did not lead to a missorting event, we performed immunocytochemistry after PMA administration to examine the vesicle population into which DAT entered. Staining with EEA1 revealed that Flot1 overexpression led to proper entry of DAT into an early endosomal compartment (Fig.2b) similar to wild-type conditions (Fig.1b).

We next determined if Flot1 was required for PMA-triggered internalization (Fig.3), using siRNA-mediated knockdown of Flot1. Depletion of endogenous Flot1 abolished PMAtriggered internalization of DAT as shown by confocal microscopy and cell surface biotinylation (Fig.3a, b). These findings implicate Flot1 as a mediator of PKC-triggered internalization of DAT. Moreover, since depletion of Flot1 did not inhibit cell surface expression of DAT, this suggested that Flot1 is not required for the trafficking of DAT to the plasma membrane. Depletion of Flot1 using siRNA had no effect on the canonical, clathrinmediated endocytosis of the transferrin receptor (TfR) (Fig.S4a), indicating that Flot1 is not required for all forms of endocytosis. Also consistent with previous reports, TfR internalization was unaffected by either PKC activation or PKC inhibition (Fig.S4b)30.

Since EAAT2 also undergoes PMA-triggered internalization and also localizes to membrane rafts, we examined whether its internalization was also dependent on Flot1. HeLa-eGFPEAAT2 demonstrated robust internalization of eGFP-EAAT2 into EAA1-positive vesicles after a 30 min exposure to $0.5 \mu \mathrm{M}$ PMA (Fig.3c). Using cell surface biotinylation, PMAtriggered internalization was re-examined after transfection with siCTRL or siFlot1 (Fig.3d). Similar to DAT, depletion of Flot1 inhibited PMA-triggered internalization of EAAT2, suggesting that these NTTs share common endocytic machinery despite being structurally distinct.

Flot1 has previously been shown to delineate a discrete set of endocytic vesicles17. We therefore next determined whether DAT and EAAT2 were trafficked into Flot1-positive vesicles. Confocal microscopy revealed that PKC-triggered internalization caused DAT to traffic into vesicles positive for Flot1 (Fig.3e) but negative for Cav1 (Fig.S3c). Further inspection at higher magnification revealed a high degree of co-localization between Flot1 and DAT (Fig.3e). Similar results were obtained for EAAT2 (Fig.S5).

Although recently implicated in endocytosis 17, Flot1 often has been used as a marker of membrane rafts. Flot1 interacts with cholesterol rich membranes through palmitoylation31, a labile and reversible post-translational modification. Flot1 is palmitoylated on a conserved 
cysteine residue, Cys34, in its highly conserved prohibitin homology domain (PHB domain)31 (Fig.4a). To examine the relevance of Flot1 palmitoylation for PKC-triggered endocytosis of DAT, we mutated Cys34 to Ala (Flot1(C34A)). As previously shown by Parton and colleagues31, the palmitoylation-deficient mutant was distributed diffusely in the cytoplasm (Fig.4b). Similar to Flot1 depletion, Flot1(C34A) overexpression did not alter basal trafficking of DAT to the plasma membrane in EM4-YFP-DAT (Fig.4b).

In stark contrast, Flot1(C34A) acted in a dominant negative manner to inhibit PMAtriggered endocytosis of DAT (Fig.4b-d). Furthermore, Gö6850-mediated inhibition was not ameliorated by overexpression of Flot1(C34A) (Fig.4c,d). Flot1 previously has been reported to act as a multimer20, 22. In cells expressing Flot1(C34A), we observed residual staining that indicated plasma membrane localization of the palmitoylation-deficient mutant (Fig.S6). We speculated that this may occur due to the multimerization of the mutant with endogenous Flot1. We therefore used co-IP to examine Flot1 multimerization. Both Flot1mRFP and Flot1(C34A)-mRFP were found in a complex with endogenous Flot1 (Fig.4e,f), suggesting that the residual membrane localization was due to Flot1(C34A)'s interaction with endogenous Flot1. Palmitoylation-deficiency, therefore, did little to alter the homomultimerization of Flot1; however since PKC-triggered endocytosis was inhibited, the resulting multimer was functionally altered. Loss of the key palmitoylation site likely destabilized the association of Flot1(C34A) to the plasma membrane, as demonstrated by its predominant expression in the cytosol.

Endocytic machinery often can be found in a complex with its cargo. We therefore determined whether Flot1 and DAT were members of a complex, and whether the interaction was modulated by Flot1 palmitoylation or PKC activation. EM4-YFP-DAT cells were transiently transfected with Flot1-mRFP or Flot1(C34A)-mRFP and were exposed to vehicle or $1 \mu \mathrm{M}$ PMA for $30 \mathrm{~min}$. Although PMA enhanced the Flot1-DAT interaction (Fig. 4f), it was significantly reduced by the C34A mutation (Fig.4f). These data indicated that palmitoylation of Flot1 was required to permit Flot1 to complex properly with DAT. Then, upon PKC activation, Flot1 multimerizes, interacts more efficiently with DAT and facilitates its internalization. Our efforts to express and purify recombinant Flot1 were unsuccessful, and thus we were unable to determine if this interaction was direct.

Since DAT and Flot1 can be found in a complex, we next examined the segregation of DAT and Flot1 after detergent extraction and density-gradient centrifugation. Sucrose flotation experiments upon fractionation with Brij58 showed that DAT co-segregated with Flot1 in low buoyancy fractions (Fig.5a-e)11, indicating that they were found in similar detergentresistant membranes, as described previously 1, 9-11. Interestingly, although knockdown of endogenous Flot1 had no detectable effect on DAT trafficking to the plasma membrane (Fig. 3a), sucrose gradients revealed that Flot 1 depletion altered the localization of DAT within the membrane: its distribution shifted to denser fractions in a manner similar to the distribution seen after cholesterol depletion by nystatin (Fig.5). In contrast, overexpression of Flot1(C34A) had no detectable impact on the distribution of DAT (Fig.5b vs. 5c,d). This supports the finding that Flot1 can establish discrete domains within the membrane independent of caveolin20, 22, suggesting a heterogeneity of membrane domains. Thus, not 
only is Flot 1 complexed to DAT, but its presence is also required to define the specific membrane domain within which DAT resides.

Previous studies have shown that the disruption of membrane rafts using methyl-betacyclodextrin $(\mathrm{MbC})$ inhibits the ability of DAT to uptake DA1, 9-11. We therefore determined if Flot1 depletion has a similar effect on DA uptake. Although we also find that MbC negatively affects DAT-mediated DA uptake in EM4-YFP-DAT cells (Table S1), over-expression of Flot1(C34A) and depletion of Flot1 did not diminish the capacity of DAT to transport $\left[{ }^{3} \mathrm{H}\right]$-DA or the more stable substrate $\left[{ }^{3} \mathrm{H}\right]$-tyramine (Fig.5f, Table I). Km and Vmax were also unaffected (Table I). We hypothesize that the difference between MbC and Flot1 depletion may lie in MbC's mechanism of action: MbC extracts cholesterol from cell membranes by binding the cholesterol within its nonpolar cyclodextrin rings and forming soluble complexes. Removal of cholesterol may impact transport through direct effects on DAT and its ability to undergo the conformational changes associated with transport, as cholesterol forms important functional interactions with many membrane proteins, including DAT32. To test this, we used another pharmacological agent, nystatin (Fig.5d, Table I). Nystatin and filipin disrupt rafts differently from MbC; they directly insert into the membrane to bind free cholesterol, thus maintaining cholesterol within the membrane. Similar to Flot1 depletion, we find that nystatin affected neither the Km nor Vmax of $\left[{ }^{3} \mathrm{H}\right]$-tyramine uptake (Table I), consistent with the lack of effect of filipin on DA uptake reported previously 10. Taken together, these data indicate that membrane raft localization of DAT is not required for DAT-mediated DA uptake. Nonetheless, the functional impact of $\mathrm{MbC}$ but not nystatin, filipin or Flot1-depletion indicates that membrane cholesterol is somehow critical for DA uptake, presumably through direct interactions with DAT.

In the presence of PMA, Flot1 more efficiently forms a complex with DAT (Fig.4f). This enhanced interaction is reminiscent of Cav1-mediated endocytosis15, 16, whereby phosphorylation of Cav1 increases its affinity for cargo. Another feature of this model is that phosphorylation of Cav1 increases its multimerization. Consistent with this hypothesis, PKC activation increased the amount of endogenous Flot1 that co-immunoprecipitated with Flot1-mRFP (Fig.6a).

The ability of Flot1 to overcome Gö6850-mediated inhibition of PKC-triggered endocytosis and to multimerize in response to PMA suggested that Flot1 is a substrate of PKC. Upon activation by PMA or other stimuli, PKC rapidly translocates from the cytosol to the plasma membrane, where it can localize within Flot1-positive membrane rafts33. We therefore examined if PKC activation led to phosphorylation of Flot1. In the initial characterization of Flot1, consensus sequences for four PKC, two tyrosine kinase and three casein kinase phosphorylation sites were identified21, 34. Although Flot1 has been shown to interact with the tyrosine kinase Src135, and to internalize in response to Fyn activation20, 36, a panel of tyrosine kinase inhibitors (PP2, PP3, Genistein or Herbimycin A) had no impact on PKCtriggered redistribution of DAT (data not shown), suggesting that PKC might phosphorylate Flot 1 directly or indirectly through another kinase. We therefore performed metabolic labeling experiments to examine the phosphorylation state of Flot1 in response to PMA (Fig. $6 \mathrm{~b}, \mathrm{S7})$. Cells transfected with Flot1-GFP were labeled with ${ }^{32} \mathrm{P}$-orthophosphate in the 
presence of vehicle, PMA or PMA + Gö6850 for $30 \mathrm{~min}$ as described in Methods. PMA led to a significant increase in Flot1 labeling that was inhibited by Gö6850, indicating that Flot1 was phosphorylated upon PKC activation.

To determine the site modified by PKC, we introduced Ser/Thr to Ala point mutations in the PKC consensus sequences of Flot1 (Fig.4a) and transfected the resulting constructs into the EM4-YFP-DAT cells. All mutants localized to the plasma membrane like wild-type Flot1. Metabolic labeling experiments revealed that the Flot1(S315A) mutant incorporated significantly less ${ }^{32} \mathrm{P}$ than wild-type Flot1 (Fig.6b). To determine if this was functionally significant, we examined PMA-triggered internalization of DAT. Although S54A-, T150Aand T229A-expressing cells showed robust internalization in response to PMA, cells expressing the S315A mutant had a significantly impaired response to PMA (Fig.6c,d; T150A- and T229A are not shown). Surface biotinylation experiments confirmed the requirement of Ser315 for PKC-triggered internalization of DAT (Fig.6d). This result strongly suggested that activated PKC triggers endocytosis of DAT by phosphorylating Ser315 in Flot1. This SXR/K motif is largely conserved across species (Fig.6e), and is adjacent to the oligomerization domain where it might possibly influence multimerization.

Since catecholaminergic cells express both Flot1 mRNA and protein37, we extended our studies to primary dopaminergic neuronal cultures to examine the role of endogenous Flot1 on endogenous DAT. We showed previously that DAT-mediated transient currents are proportional to the number of transporters on the cell-surface2. Thus, to determine whether Flot1 is also required for internalization of endogenous DAT, we used an electrophysiological approach to measure DAT in the plasma membrane at the single cell level. Midbrain DA neurons were cultured from mice expressing RFP driven by the tyrosine hydroxylase $(\mathrm{TH})$ promoter as described previously38. Neurons were transduced at 5 days in vitro (DIV5) with lentivirus carrying turboGFP co-expressed with either shRNA against Flot1 (shFlot1) or a non-silencing shRNA control (NonSil) and recorded 5 days later (Fig. 7a, S8). Only cells co-expressing both red and green fluorescence were examined. Under control conditions, DAT-mediated transient currents, which reflect DAT plasma membrane expression, decreased significantly in the presence of PMA, indicative of a PKC-triggered reduction of endogenous DAT cell surface expression in neurons (Fig.1, 7a). Importantly, knockdown of Flot1 (shFlot1) blocked the ability of PMA to reduce DAT cell surface expression as measured by transient currents (Fig.7a bottom panel). This confirmed that Flot1 was required for PKC-triggered endocytosis of endogenous DAT and, importantly, indicated that the loss of Flot1 has functional consequences in neurons.

Amphetamine (AMPH), a substrate of DAT, exerts its psychostimulants effects by elevation of extracellular DA through DAT-mediated DA efflux39. This process has been shown to be regulated by PKC and CamKIIalpha38, 40. To determine if AMPH-mediated reverse transport of DA in primary DA neurons is also regulated by DAT localization and therefore dependent on Flot1, we recorded DAT-mediated DA efflux using amperometry combined with patch clamp in the whole-cell configuration. This technique permits the recording of DAT-mediated DA efflux under constant voltage conditions38. Administration of $10 \mu \mathrm{M}$ AMPH to the bath increased AMPH-induced DA efflux in a voltage-dependent manner (Fig. 7b). Knockdown of Flot1 led to a significant inhibition of AMPH-induced DA efflux 
compared to non-silencing control, suggesting that Flot1-mediated localization of DAT in membrane rafts is required for efficient AMPH-induced reverse transport of DA.

\section{DISCUSSION}

We have identified Flot1 as a requisite intermediary of PKC-triggered endocytosis of two structurally distinct NTTs found in membrane rafts, DAT and EAAT2. Further examination revealed that Flot1 is also a critical scaffold that maintains DAT in a cholesterol-rich environment within the plasma membrane. Interestingly, the membrane environment does not impact DAT-mediated uptake of DA, but instead is required for AMPH-induced reverse transport of DA. In summary, this study identifies DAT and EAAT2 as cargo for Flot1mediated endocytosis, and also demonstrates that Flot1 is likely to play an important role in synaptic function and homeostasis.

Metabolic labeling experiments demonstrated that PKC activation increased the phosphorylation of Flot1 on residue Ser315 (Fig.6). In turn, Flot1 increased its ability to homomultimerize (Fig.4f, 6a) and to interact with DAT (Fig.4f), supporting the model that Flot1-mediated endocytosis may be regulated similarly to caveolin-mediated endocytosis16, 22. Sucrose gradient analysis also indicates that like caveolin, Flot1 may form unique, stable scaffolds to form subdomains within the plasma membrane, creating a microenvironment in which key components can organize. How multimerization of Flot1 influences its cargo selection, and what other cargo may be specific for Flot1, remain to be studied. Moreover, although Flot1 previously has been shown to interact with src kinases35, the adaptor protein CAP41, as well as other proteins 23 , it is not yet certain what other adaptor proteins also may be required for internalization of DAT and EAAT2. Further examination of these questions will not only permit insights into DAT regulation and function, but will also permit greater insight into the endocytic machinery involved.

A role for DAT ubiquitination in its PKC-mediated-, clathrin- and epsin-dependent internalization and subsequent degradation has previously been reported by Sorkin and colleagues42, 43. Moreover, endocytosis studies in PAE cells43 and LLC-PK1 cells 10 after nystatin and $\mathrm{MbC}$ treatment respectively, showed that depletion of cholesterol from membranes does not disrupt PKC-triggered DAT internalization, suggesting that only the transporter present outside of membrane rafts is trafficked. Although different cell lines have been used in these studies, and differences in constitutive endocytosis levels should be considered, it is not yet clear whether our findings with Flot1 represent an independent trafficking pathway or a Flot1-dependent form of clathrin-mediated DAT endocytosis. Although it is conceivable that Flot1 organizes DAT for clathrin-mediated endocytosis as a novel adaptor protein, Glebov et al17 reported that Flot1-mediated internalization of cargo is both clathrin- and Cav1-independent. Moreover, the highly homologous Flot2 also internalizes in a clathrin-independent manner 20.

A likely scenario is that DAT (and possibly EAAT2 as well) may be internalized by different pathways, leading to different final destinations. In the case of the TGF-beta receptor44, caveolin-dependent internalization leads to rapid receptor degradation, whereas clathrin-dependent internalization promotes TGF-beta recycling and signaling. Similarly, 
DAT may be sorted by Flot1 into a recycling pool and by clathrin into a degradation pool or vice versa. Clathrin- and epsin-mediated internalization and the endocytosis of proteins associated in membrane rafts have been considered to be essentially mutually exclusive 45 , and it is possible that one means by which this sorting is achieved is by the membrane localization of DAT within Flot1-positive and possibly other microdomains. Sorting also may depend upon the strength and duration of the trigger. For example, sustained exposure to low concentrations of PMA have been shown previously to lead to internalization of a GPi-linked cargo, CD5946. Although the depletion of Flot1 inhibits DAT internalization after $1 \mu \mathrm{M}$ PMA for $30 \mathrm{~min}$, preliminary evidence indicates that DAT-positive intracellular vesicles can be observed after $0.1 \mu \mathrm{M}$ PMA exposure for $2 \mathrm{hr}$ (data not shown). Additional studies examining the kinetics of internalization, recycling and degradation of DAT and mechanisms of Flot1-mediated endocytosis will help define the relationship between the roles of clathrin and Flot1. Moreover, since endogenous DAT in both primary cultures (Fig. 1c) and slice (Fig. 1d) also can internalize in a PKC-dependent manner, studies in endogenous systems will also shed light on the cell surface regulation of endogenous DAT.

An unexpected finding of our studies is that Flot1 is required to maintain DAT in membrane rafts. In light of these findings, a step-wise transition from a membrane microdomain to a clathrin-dependent endocytic event outside of membrane rafts may be possible. A similar model has been reported for the tetanus neurotoxin fragment (TeNT HC) receptor, which is maintained in lipid microdomains by binding to the protein GD1b. Dissociation from GD1b permits TeNT HC receptor to exit from the membrane raft and internalize in a clathrindependent (however epsin-independent) fashion. Flot1 may similarly 'hold' DAT in membrane rafts, although in immunoprecipitation studies PKC activation increases rather than decreases the interaction between Flot1 and DAT (Fig. 4f), and thus different regulatory mechanisms may be at play.

Disruption of membrane rafts through cholesterol depletion using $\mathrm{MbC}$ has been shown to affect DAT10 and the structurally similar SERT9, 11 transport activity in heterologous systems. Consistent with previous reports, we have found that $\mathrm{MbC}$ impairs DAT transport activity (Table S1); however we also find that Flot1 depletion and nystatin treatment do not (Table 1). This indicates that the impact of $\mathrm{MbC}$ on transport activity does not result only from disruption of rafts, and the presence of membrane cholesterol is important for DAT function. Although DAT transport activity is unaffected, the Flot1-dependent membrane microdomain environment in neurons is quite important for AMPH-induced DA efflux in DA neurons. The loss of DA efflux after Flot1 depletion suggests that the behavioral effects of AMPH would be inhibited in the absence of Flot1. We are currently testing this hypothesis in different animal models to establish whether there is a link between localization of DAT in Flot1-positive cholesterol-rich membrane microdomains and the behavioral consequences due to AMPH-mediated DA efflux.

\section{Methods Section}

\section{Reagents}

Antibodies: Becton Dickenson (Flot1, EEA1), Abcam (Flot1, Cav1, GFP), Invitrogen (hTfR), Roche (GFP), Santa Cruz Biotechnology (Flot1) and Clontech (DsRed (RFP). 
Invitrogen (Alexa 633 CTX B subunit, AlexaFluor secondaries, Lipofect- and OligofectAMINE), Sigma (PMA), Calbiochem (Gö6850), Miltenyi Biotec (anti-GFP beads), Pierce (sulfo-NHS-LC-biotin, EZ-Link Sulfo-NHS-SS-Biotin, Secondary antibodies, ImmunoPure streptavidin beads), Integrated DNA Technology (siCtrl, siCav1, siFlot1_1, siFlot1_2), Santa Cruz Biotechnology (siFlot1), Open Biosystems (shRNA, lentiviral kit).

\section{Flotillin-1 constructs}

hFlot1 was purchased from Origene. hFlot1 was subcloned into pClontech monomeric dsRED (mRFP) or pcDNA3.1(+). Site-directed mutagenesis was performed using QuikChange.

\section{Transfections/Transductions}

siRNAs were used singly at a final concentration of $10 \mathrm{nM}$, or as a mix of two siRNAs as described 17 and transfected into cells using OligofectAMINE. Lentiviri carrying shFlot1 or shNonSil with turboGFP were created per manufacturer's instructions. Primary neurons were transduced on DIV5 with MOI of 8.

\section{Cell culture}

Production of stable cell lines: cDNAs clones expressing DAT, SERT and EAAT2 were subcloned by PCR into pEGFP-C1 and pEYFP-C1 then pIREShyg3. Cells were transfected, then individual clones were selected using 400 or $200 \mu \mathrm{g} / \mathrm{ml}$ hygromycin for HeLa and HEK293 cells, respectively.

EM4-YFP-DAT were created as described previously2, 47. EM4 cells are human embryonic kidney 293 cells modified to increase their adherence to tissue culture plastic29.

Primary mesencephalic dopaminergic neurons were generated as described 38.

\section{Transporter internalization assay}

Cell lines: To initiate endocytosis, cells were rinsed twice with PBS and once with KrebsRinger's-HEPES (KRH) then incubated with $\mathrm{KRH}+\mathrm{PMA}$ for $30 \mathrm{~min}$ at $37^{\circ} \mathrm{C}$. Internalization was stopped with cold KRH. Gö6850 was administered in 30min prior to PMA administration, and maintained for the duration of the experiment.

Primary cultures: Neurons were incubated in $\mathrm{KRH}+$ glucose for $1 \mathrm{hr}$ at $37^{\circ} \mathrm{C}$. Cultures were treated with 30 min PMA, and then fixed.

Brain slices: Mice were handled using Vanderbilt University IACUC approved procedures. 6- to 10-week-old mice were anesthetized with isoflourane and rapidly decapitated. Brains were chilled in $4^{\circ} \mathrm{C}$, oxygenated sucrose solution (sucrose $210 \mathrm{mM} ; \mathrm{NaCl} 20 \mathrm{mM} ; \mathrm{KCl}$ $2.5 \mathrm{mM} ; \mathrm{MgCl}_{2} 1 \mathrm{mM} ; \mathrm{NaH}_{2} \mathrm{PO}_{4} \bullet \mathrm{H} 2 \mathrm{O} 1.2 \mathrm{mM}$ ), then sliced into $300 \mu \mathrm{m}$ coronal slices. Slices were collected then washed twice with oxygenated artificial cerebral spinal fluid (ACSF) ( $\mathrm{NaCl} 125 \mathrm{mM}, \mathrm{KCl} 2.5 \mathrm{mM}, \mathrm{NaH}_{2} \mathrm{PO}_{4} \bullet \mathrm{H} 2 \mathrm{O} 1.2 \mathrm{mM}, \mathrm{MgCl}_{2} 1 \mathrm{mM}, \mathrm{CaCl}_{2} \bullet 2 \mathrm{H}_{2} \mathrm{O} 2 \mathrm{mM}$ ). Slices were treated for $1 \mathrm{hr}$ with PMA then cell surface biotinylated. 


\section{Cell surface biotinylation assays}

Stable cell lines: Cell surface proteins were biotinylated with the membrane-impermeant reagent Sulfo-NHS-LC-Biotin in PBS containing $1 \mathrm{mM} \mathrm{MgCl} 2,0.1 \mathrm{mM} \mathrm{CaCl}_{2}\left(\mathrm{PBS}^{2+}\right)$. Cells were subsequently washed in $\mathrm{PBS}^{2+}$, lysed in a $1 \% \mathrm{Tx}-100$ buffer containing protease inhibitors, and cleared by centrifugation. Biotinylated proteins were separated from nonbiotinylated by $4{ }^{\circ} \mathrm{C}$, overnight incubation with equilibrated NeutrAvidin agarose beads.

Brain slices: Slices were incubated with $4^{\circ} \mathrm{C}$ ACSF solution containing $1 \mathrm{mg} / \mathrm{mL}$ EZ-Link Sulfo-NHS-SS-Biotin for $45 \mathrm{~min}$. After biotin incubation, the slices were rinsed quickly twice and the reaction was quenched by two $20 \mathrm{~min}$ washes in $4^{\circ} \mathrm{C}$ oxygenated ACSF + glycine. Following quenching, slices were lysed in buffer B (25mM HEPES, 150mM $\mathrm{NaCl}, 2 \mathrm{mM} \mathrm{Na}_{3} \mathrm{VO}_{4}, 2 \mathrm{mM} \mathrm{NaF}$ plus a cocktail of protease inhibitors) $+1 \% \mathrm{Tx}-100$, then centrifuged at $17,000 \times \mathrm{g}$ for $30 \mathrm{~min}$ at $4^{\circ} \mathrm{C}$. Biotinylated proteins were isolated using ImmunoPure immobilized streptavidin beads overnight at $4^{\circ} \mathrm{C}$ with agitation. Beads were washed 3 times with Buffer B $+0.1 \%$ Tx-100 and biotinylated proteins were eluted in $50 \mu \mathrm{L}$ of $2 \mathrm{X}$ SDS-PAGE sample loading buffer at $95^{\circ} \mathrm{C}$ and probed for DAT48.

\section{Immunofluorescence (IF)}

Stable cell lines: Cells were fixed either with $4 \%$ paraformaldehyde for $5 \mathrm{~min}$ at RT, or with methanol for $20 \mathrm{~min}$ at $-20^{\circ} \mathrm{C}$ (anti-Cav1, anti-Flot1 IF). Cells were washed 3x with PBS (or PBS+0.01\% Triton X-100 to permeabilize) and blocked with 5\% normal goat serum in PBS. Primary antibodies were diluted in blocking solution (EEA1, 1:1000; Cav1, $1 \mu \mathrm{g} / \mathrm{ml}$; Flot1 1:100), and incubated overnight at $4^{\circ} \mathrm{C}$. Cells were washed $3 \mathrm{x}$ with PBS, and incubated with secondary antibody for $1 \mathrm{hr}$ at RT.

Primary neurons: Permeabilized cultures were blocked with PBS-5\% BSA-0.05\% Tween 20 (PBSBT), treated with anti-DAT antibody in PBSBT for $4 \mathrm{hrs}$ at RT or overnight at $4{ }^{\circ} \mathrm{C}$. Cultures were washed with PBSBT, incubated in secondary antibodies conjugated to Alexa 568 , and imaged.

\section{Microscopy and image analysis}

Stable cell lines: Cells were grown on glass (HeLa) or poly-D-lysine coated (HEK-293, EM4) coverslips. After treatments, cells were fixed for $10 \mathrm{~min}$ in 4\% PFA. '\% Cell with Internalization' was calculated as cells considered positive for internalization (YFP-DAT co-localized to EEA1-positive endosomes) after 30min PMA. Images were acquired using the Leica TCS SP 2 confocal microscope at $40 \times$ or $63 \times$ magnification and the accompanying software package. Data analysis was performed using National Institutes of Health Image 4.0 (http://rsb.info.nih.gov/nih-image/).

Primary neurons: Cells were imaged at 16 bits on an Olympus FLUOVIEW FV1000, 60X NA 1.42 lens. Data were collected using 16 bit data. The maximal brightness was established at the surface and internal compartment by thresholding. Background values were determined and subtracted. The internalization index was calculated by dividing the maximal brightness of the interior by the maximal brightness of the surface. 


\section{Transferrin uptake assay}

Stable cell lines were incubated in $\mathrm{KRH}$ medium with $0.2 \% \mathrm{BSA}$ for $1 \mathrm{hr}$ at $37^{\circ} \mathrm{C}$ with $5.0 \%$ $\mathrm{CO}_{2}$ to remove surface-bound transferrin. The cells were washed twice in ice cold $\mathrm{KRH}$ then incubated in $5 \mu \mathrm{g} / \mathrm{ml}$ of Alexa 633 -conjugated transferrin at $4^{\circ} \mathrm{C}$. Internalization was initiated by warming the cells to $37^{\circ} \mathrm{C}$ for the times indicated and was stopped by washing repeatedly with ice-cold PBS.

\section{Sucrose flotation gradients}

EM4-YFP-DAT cells were washed twice with ice-cold PBS and lysed in $1 \mathrm{ml}$ HEPESbuffered saline containing $1 \% \mathrm{w} / \mathrm{v}$ Brij 58 and protease inhibitors for $30 \mathrm{~min}$ at $4{ }^{\circ} \mathrm{C}$. Lysates were homogenized with 15 passages through a 20 -gauge needle, transferred to a minifuge tube and centrifuged at $4{ }^{\circ} \mathrm{C}$ for $10 \mathrm{~min}$ at $830 \times \mathrm{g}$. $1 \mathrm{ml}$ of cell supernatant was mixed with an equal volume of $80 \%$ (w/v) sucrose in HEPES buffer, transferred into an ultracentrifuge tube, then carefully overlaid with $1 \mathrm{ml}$ of $30 \%$ (w/v) sucrose in HEPES buffer, $1 \mathrm{ml}$ of $15 \%$ $(\mathrm{w} / \mathrm{v})$ sucrose in HEPES buffer and finally with $0.75 \mathrm{ml}$ of $5 \%(\mathrm{w} / \mathrm{v})$ sucrose in HEPES buffer. The tube was placed in a cooled SW55Ti swinging-bucket rotor, and centrifugation was carried out in a Beckman ultracentrifuge at $4^{\circ} \mathrm{C}$ for $20 \mathrm{hr}$ at $45,000 \mathrm{rpm}$.

\section{DA and tyramine uptake assay}

DA uptake on EM4-YFP-DAT: Cells were placed on ice and washed 3 times with ice-cold KRH buffer after PMA internalization. Cells were then administered $\left[{ }^{3} \mathrm{H}\right] \mathrm{DA}$ and $3 \mu \mathrm{M}$ cold DA in KRH buffer and returned to the incubator for $1 \mathrm{~min}$ at $37^{\circ} \mathrm{C}$. To terminate uptake, cells were quickly transferred to ice and the DA-containing buffer was aspirated. Cells were washed 3 times with ice cold KRH buffer. Cells were permeabilized using 0.1\% Tx-100 (in PBS), then measured for radioactivity. To determine non-specific uptake, a subset of cells were also exposed to $25 \mathrm{nM}$ GBR12935. To obtain Km and Vmax values, $\left[{ }^{3} \mathrm{H}\right]$ tyramine uptake competition assays were also performed as previously described 49 .

\section{Immunoprecipitation and immunoblotting}

Cell lysis with 50mM TrisHCl, pH7.4, 150mM NaCl, 5mM EDTA, 1X protease inhibitor cocktail (Roche) and 1\% Tx-100 on ice for 30min. All solutions were pre-chilled and all steps in the lysis procedure were performed on ice. After pre-clearing with $100 \mu \mathrm{L}$ of protein A agarose slurry with $1 \mathrm{~mL}$ of protein, $1 \mu \mathrm{g} / \mathrm{mL}$ of lysate were incubated overnight with either: anti-mRFP or anti-Flot 1 followed by protein A-agarose bead slurry $(50 \mu \mathrm{L}$ of a $50 \%$ suspension, Invitrogen) $1 \mathrm{hr}$ at $4^{\circ} \mathrm{C}$ with gentle agitation; anti-3xFLAG agarose beads (Sigma); or with anti-GFP antibody conjugated to magnetic beads (Miltenyi). Agarose beads were collected in batch by gentle centrifugation, washed with buffer $(150 \mathrm{mM} \mathrm{NaCl}, 50 \mathrm{mM}$ TrisHCl, $1 \%$ NP40, $0.5 \%$ Na deoxycholate and $0.1 \%$ SDS ) and resuspended in $2 \times$ SDS loading buffer and ran on SDS-PAGE. Magnetic beads were collected on a magnetic column, washed using similar conditions and eluted in $2 \times$ sample buffer.

Proteins were resolved by SDS-PAGE and detection by Western blot as previously described50. 
Antibody concentrations were as follows: Anti-DsRed (mRFP): 1:8000, anti-GFP: 1:1000, anti-Flot1: 1:250, anti-hTfR: $1 \mu \mathrm{g} / \mu \mathrm{L}$, anti-EEA1: 1:1000, anti-Cav1: $1 \mu \mathrm{g} / \mu \mathrm{L}$.

\section{In vivo radio labeling and total cellular protein extraction}

EM4-YFP-DAT cells were transfected with pFlot-GFP. Next, cells were labeled with $0.5 \mathrm{mCi} / \mathrm{ml}$ of ${ }^{32} \mathrm{Pi}$ (orthophosphate) in phosphate-free MEM supplemented with $10 \%$ dialyzed FBS from 40 to $48 \mathrm{hr}$ post-infection. Cells were then either treated or as described. Cells were harvested by scraping and were resuspended in $0.5 \mathrm{ml}$ of lysis buffer $(50 \mathrm{mM}$ Tris-HCl, pH 8.0, 150mM NaCl, 20mM EDTA, $1 \%$ Triton X-100, $10 \%$ glycerol and protease inhibitor cocktail -Protease Arrest Reagent, Calbiochem). Cells were incubated in an ice bath for $1 \mathrm{hr}$ and centrifuged at $10,000 \times \mathrm{g}$ for $10 \mathrm{~min}$ at $4{ }^{\circ} \mathrm{C}$. The resulting supernatants were used as total cellular extracts. The extracts were immunoprecipitated using anti-GFP beads (Miltenyi Biotech). The immunoprecipitates were electrophoresed in a 10\% SDSPAGE gel. The resulting gel was fixed in $7.5 \%$ acetic acid $/ 20 \%$ methanol in water for $30 \mathrm{~min}$, then incubated for $15 \mathrm{~min}$ in $4.5 \%$ glycerol $/ 20 \%$ methanol in water and dried or transferred to PVDF then probed for Flot1. The gel was exposed to Kodak Biomax X-ray film with a Fisher Biotech L Plus screen at $-80^{\circ} \mathrm{C}$ for $24 \mathrm{hr}$.

\section{Electrophysiological analysis of DA neurons}

Only cells co-expressing both red (RFP expression driven by tyrosine hydroxylase promoter) and green (shFlot1 or shNonSil) fluorescence were utilized in electrophysiological assays.

The bath solution for both experiments contained (in $\mathrm{mM}$ ) $146 \mathrm{NaCl}, 5 \mathrm{KCl}, 5 \mathrm{HEPES}, 2.5$ $\mathrm{CaCl}_{2}, 1.2 \mathrm{MgCl}_{2}$, and 30 Dextrose ( $\mathrm{pH}$ 7.35). A programmable puller (model P-2000, Sutter Instruments) was used to fabricate quartz recording pipettes with a resistance of $7 \mathrm{M} \Omega$.

Whole cell currents: Neuronal cultures were washed twice with the bath solution. Sulpiride $(1 \mu \mathrm{M})$ was added to the bath solution to inhibit D2 receptor-mediated regulation of DAT. The pipette solution for recording contained (in $\mathrm{mM}$ ): $108 \mathrm{CsCl}, 30 \mathrm{NaCl}, 2 \mathrm{MgCl}_{2}, 1$ EGTA, $0.1 \mathrm{CaCl}_{2}$, 2 Mg-ATP, 2 Na-GTP, 10 HEPES, and 30 dextrose (pH 7.35). Wholecell currents were recorded using an Axopatch 200B with a low pass Bessel filter set at $5,000 \mathrm{~Hz}$. Current-voltage relationships were generated by stepping the membrane voltage from a holding potential of $-20 \mathrm{mV}$ to voltages between -140 and $100 \mathrm{mV}$ in $20 \mathrm{mV}$ increments for $1 \mathrm{sec}$. DAT-mediated currents were obtained by subtracting the current trace recorded in the presence of cocaine at the end of each experiment from the current traces recorded in the control and PMA-treated conditions. The DAT transient charge (Q) movement in response to a voltage step, was obtained by integrating either the "on" or the "off" of the relaxation component of the current during the voltage steps. Time-dependent changes in Q were used to evaluate hDAT cell surface expression as described previously38.

Amperometric currents (as described38): Neurons were washed twice with the bath solution containing. The pipette solution for whole cell recording contained (in $\mathrm{mM}$ ) $108 \mathrm{KCl}, 30$ $\mathrm{NaCl}, 2 \mathrm{MgCl}_{2}, 0.1 \mathrm{CaCl}_{2}, 1.1$ EGTA, 10 HEPES, $4 \mathrm{MgATP}$, and 30 dextrose (pH 7.35) plus 2 DA. To measure amperometric currents, a carbon fiber electrode was simultaneously attached to the plasma membrane of the cell and held at $+700 \mathrm{mV}$, greater than the oxidizing 
potential of DA. Whole cell and amperometric currents were recorded using an Axopatch 200B with the amperometric currents low pass filtered at $100 \mathrm{~Hz}$. Amperometric currentvoltage relationship was generated using a voltage step (1sec) protocol ranging from $0 \mathrm{mV}$ to $100 \mathrm{mV}$ separated by $20 \mathrm{mV}$ from a $-60 \mathrm{mV}$ holding potential. This protocol first was performed before drug application (control) then repeated after 3 min of $10 \mu \mathrm{M} \mathrm{AMPH}$ application to the bath followed by addition of $10 \mu \mathrm{M}$ cocaine for $5 \mathrm{~min}$ to the bath. AMPHinduced hDAT currents were defined as the current recorded in the presence of AMPH, minus the current recorded after the addition of cocaine to the bath with AMPH still present.

\section{Statistical Analyses and Image preparation}

Statistical significance was determined using ANOVA unless otherwise noted. For variables that showed significant ANOVA interactions between variables, post hoc tests were performed with Fisher's protected least significant difference (PLSD) test for multiple comparisons. All calculations were performed using Statview 5.0 (SAS Institute). Additional statistics for all figures can be found under 'Statistical Analyses' in Supporting Materials. After image acquisition, images were cropped and figures prepared using Adobe Create Suite 3.

\section{Supplementary Material}

Refer to Web version on PubMed Central for supplementary material.

\section{Acknowledgements}

We would like to thank Caline Karam, Tom Melia, Andrea Pizzo, Harald Stenmark and Hideki Yano for critical reading of this manuscript. This work was supported by NINDS Research grant RO1 NS050199 and the Parkinson's Disease Foundation (A.Y.) and NIDA Research grant PO1 DA12408 (A.G. and J.A.J.), DA13975 (A.G.), K05 DA022413 (J.A.J.) and DA13147 (R.A.V.).

\section{Reference}

1. Melikian HE. Neurotransmitter transporter trafficking: endocytosis, recycling, and regulation. Pharmacol Ther. 2004; 104:17-27. [PubMed: 15500906]

2. Kahlig KM, Javitch JA, Galli A. Amphetamine regulation of dopamine transport. Combined measurements of transporter currents and transporter imaging support the endocytosis of an active carrier. J Biol Chem. 2004; 279:8966-8975. [PubMed: 14699142]

3. Gonzalez MI, Susarla BT, Robinson MB. Evidence that protein kinase Calpha interacts with and regulates the glial glutamate transporter GLT-1. J Neurochem. 2005; 94:1180-1188. [PubMed: 16045453]

4. Kalandadze $\mathrm{A}, \mathrm{Wu} \mathrm{Y}$, Robinson $\mathrm{MB}$. Protein kinase $\mathrm{C}$ activation decreases cell surface expression of the GLT-1 subtype of glutamate transporter. Requirement of a carboxyl-terminal domain and partial dependence on serine 486. J Biol Chem. 2002; 277:45741-45750. [PubMed: 12324450]

5. Zhou J, Sutherland ML. Glutamate transporter cluster formation in astrocytic processes regulates glutamate uptake activity. J Neurosci. 2004; 24:6301-6306. [PubMed: 15254085]

6. Guillet BA, et al. Differential regulation by protein kinases of activity and cell surface expression of glutamate transporters in neuron-enriched cultures. Neurochem Int. 2005; 46:337-346. [PubMed: 15707698]

7. Simons K, Ikonen E. Functional rafts in cell membranes. Nature. 1997; 387:569-572. [PubMed: 9177342] 
8. Pike LJ. Rafts defined: a report on the Keystone Symposium on Lipid Rafts and Cell Function. J Lipid Res. 2006; 47:1597-1598. [PubMed: 16645198]

9. Adkins EM, et al. Membrane mobility and microdomain association of the dopamine transporter studied with fluorescence correlation spectroscopy and fluorescence recovery after photobleaching. Biochemistry. 2007; 46:10484-10497. [PubMed: 17711354]

10. Foster JD, Adkins SD, Lever JR, Vaughan RA. Phorbol ester induced trafficking-independent regulation and enhanced phosphorylation of the dopamine transporter associated with membrane rafts and cholesterol. J Neurochem. 2008; 105:1683-1699. [PubMed: 18248623]

11. Magnani F, Tate CG, Wynne S, Williams C, Haase J. Partitioning of the serotonin transporter into lipid microdomains modulates transport of serotonin. J Biol Chem. 2004; 279:38770-38778. [PubMed: 15226315]

12. Escartin C, et al. Ciliary neurotrophic factor activates astrocytes, redistributes their glutamate transporters GLAST and GLT-1 to raft microdomains, and improves glutamate handling in vivo. J Neurosci. 2006; 26:5978-5989. [PubMed: 16738240]

13. Butchbach ME, Tian G, Guo H, Lin CL. Association of excitatory amino acid transporters, especially EAAT2, with cholesterol-rich lipid raft microdomains: importance for excitatory amino acid transporter localization and function. J Biol Chem. 2004; 279:34388-34396. [PubMed: 15187084]

14. Doherty GJ, McMahon HT. Mechanisms of endocytosis. Annu Rev Biochem. 2009; 78:857-902. [PubMed: 19317650]

15. Bauer M, Pelkmans L. A new paradigm for membrane-organizing and -shaping scaffolds. FEBS Lett. 2006; 580:5559-5564. [PubMed: 16996501]

16. Pelkmans L, Burli T, Zerial M, Helenius A. Caveolin-stabilized membrane domains as multifunctional transport and sorting devices in endocytic membrane traffic. Cell. 2004; 118:767780. [PubMed: 15369675]

17. Glebov OO, Bright NA, Nichols BJ. Flotillin-1 defines a clathrin-independent endocytic pathway in mammalian cells. Nat Cell Biol. 2006; 8:46-54. [PubMed: 16341206]

18. Damm EM, et al. Clathrin- and caveolin-1-independent endocytosis: entry of simian virus 40 into cells devoid of caveolae. J Cell Biol. 2005; 168:477-488. [PubMed: 15668298]

19. Benlimame N, Le PU, Nabi IR. Localization of autocrine motility factor receptor to caveolae and clathrin-independent internalization of its ligand to smooth endoplasmic reticulum. Mol Biol Cell. 1998; 9:1773-1786. [PubMed: 9658170]

20. Stuermer CA. Reggie/flotillin and the targeted delivery of cargo. J Neurochem. 2011; 116:708713. [PubMed: 21214550]

21. Galbiati F, et al. Identification, sequence and developmental expression of invertebrate flotillins from Drosophila melanogaster. Gene. 1998; 210:229-237. [PubMed: 9573373]

22. Frick M, et al. Coassembly of flotillins induces formation of membrane microdomains, membrane curvature, and vesicle budding. Curr Biol. 2007; 17:1151-1156. [PubMed: 17600709]

23. Morrow IC, Parton RG. Flotillins and the PHB domain protein family: rafts, worms and anaesthetics. Traffic. 2005; 6:725-740. [PubMed: 16101677]

24. Zacharias DA, Violin JD, Newton AC, Tsien RY. Partitioning of lipid-modified monomeric GFPs into membrane microdomains of live cells. Science. 2002; 296:913-916. [PubMed: 11988576]

25. Chang MY, et al. Protein kinase C-mediated functional regulation of dopamine transporter is not achieved by direct phosphorylation of the dopamine transporter protein. J Neurochem. 2001; 77:754-761. [PubMed: 11331404]

26. Granas C, Ferrer J, Loland CJ, Javitch JA, Gether U. N-terminal truncation of the dopamine transporter abolishes phorbol ester- and substance $\mathrm{P}$ receptor-stimulated phosphorylation without impairing transporter internalization. J Biol Chem. 2003; 278:4990-5000. [PubMed: 12464618]

27. Wang L, et al. Caveolin-1 does not affect SR-BI-mediated cholesterol efflux or selective uptake of cholesteryl ester in two cell lines. J Lipid Res. 2003; 44:807-815. [PubMed: 12562842]

28. Wharton J, Meshulam T, Vallega G, Pilch P. Dissociation of insulin receptor expression and signaling from caveolin-1 expression. J Biol Chem. 2005; 280:13483-13486. [PubMed: 15699039] 
29. Robbins AK, Horlick RA. Macrophage scavenger receptor confers an adherent phenotype to cells in culture. BioTechniques. 1998; 25:240-244. [PubMed: 9714883]

30. Buckley KM, Melikian HE, Provoda CJ, Waring MT. Regulation of neuronal function by protein trafficking: a role for the endosomal pathway. J Physiol. 2000; 525(Pt 1):11-19. [PubMed: 10811720]

31. Morrow IC, et al. Flotillin-1/reggie-2 traffics to surface raft domains via a novel golgi-independent pathway. Identification of a novel membrane targeting domain and a role for palmitoylation. J Biol Chem. 2002; 277:48834-48841. [PubMed: 12370178]

32. Hong WC, Amara SG. Membrane cholesterol modulates the outward-facing conformation of the dopamine transporter and alters cocaine binding. J Biol Chem. 2010

33. Becart S, et al. Intracytoplasmic domains of MHC class II molecules are essential for lipid-raftdependent signaling. J Cell Sci. 2003; 116:2565-2575. [PubMed: 12766188]

34. Bickel PE, et al. Flotillin and epidermal surface antigen define a new family of caveolae-associated integral membrane proteins. J Biol Chem. 1997; 272:13793-13802. [PubMed: 9153235]

35. Kato N, Nakanishi M, Hirashima N. Flotillin-1 regulates IgE receptor-mediated signaling in rat basophilic leukemia (RBL-2H3) cells. J Immunol. 2006; 177:147-154. [PubMed: 16785509]

36. Riento K, Frick M, Schafer I, Nichols BJ. Endocytosis of flotillin-1 and flotillin-2 is regulated by Fyn kinase. J Cell Sci. 2009; 122:912-918. [PubMed: 19258392]

37. Jacobowitz DM, Kallarakal AT. Flotillin-1 in the substantia nigra of the Parkinson brain and a predominant localization in catecholaminergic nerves in the rat brain. Neurotox Res. 2004; 6:245257. [PubMed: 15545008]

38. Fog JU, et al. Calmodulin kinase II interacts with the dopamine transporter C terminus to regulate amphetamine-induced reverse transport. Neuron. 2006; 51:417-429. [PubMed: 16908408]

39. Sulzer D, Sonders MS, Poulsen NW, Galli A. Mechanisms of neurotransmitter release by amphetamines: a review. Prog Neurobiol. 2005; 75:406-433. [PubMed: 15955613]

40. Chen R, et al. Protein kinase Cbeta is a critical regulator of dopamine transporter trafficking and regulates the behavioral response to amphetamine in mice. J Pharmacol Exp Ther. 2009; 328:912920. [PubMed: 19098163]

41. Baumann CA, et al. CAP defines a second signalling pathway required for insulin-stimulated glucose transport. Nature. 2000; 407:202-207. [PubMed: 11001060]

42. Miranda M, Dionne KR, Sorkina T, Sorkin A. Three ubiquitin conjugation sites in the amino terminus of the dopamine transporter mediate protein kinase $\mathrm{C}$-dependent endocytosis of the transporter. Mol Biol Cell. 2007; 18:313-323. [PubMed: 17079728]

43. Sorkina T, Hoover BR, Zahniser NR, Sorkin A. Constitutive and protein kinase C-induced internalization of the dopamine transporter is mediated by a clathrin-dependent mechanism. Traffic. 2005; 6:157-170. [PubMed: 15634215]

44. Di Guglielmo GM, Le Roy C, Goodfellow AF, Wrana JL. Distinct endocytic pathways regulate TGF-beta receptor signalling and turnover. Nat Cell Biol. 2003; 5:410-421. [PubMed: 12717440]

45. Deinhardt K, Berninghausen O, Willison HJ, Hopkins CR, Schiavo G. Tetanus toxin is internalized by a sequential clathrin-dependent mechanism initiated within lipid microdomains and independent of epsin1. J Cell Biol. 2006; 174:459-471. [PubMed: 16880274]

46. Idkowiak-Baldys J, Becker KP, Kitatani K, Hannun YA. Dynamic sequestration of the recycling compartment by classical protein kinase C. J Biol Chem. 2006; 281:22321-22331. [PubMed: 16751194]

47. Sen N, Shi L, Beuming T, Weinstein H, Javitch JA. A pincer-like configuration of TM2 in the human dopamine transporter is responsible for indirect effects on cocaine binding. Neuropharmacology. 2005; 49:780-790. [PubMed: 16216288]

48. Vaughan RA, Kuhar MJ. Dopamine transporter ligand binding domains. Structural and functional properties revealed by limited proteolysis. J Biol Chem. 1996; 271:21672-21680. [PubMed: 8702957]

49. Khoshbouei $\mathrm{H}$, et al. N-terminal phosphorylation of the dopamine transporter is required for amphetamine-induced efflux. PLoS biology. 2004; 2:E78. [PubMed: 15024426]

50. Filimonenko M, et al. The selective macroautophagic degradation of aggregated proteins requires the PI3P-binding protein Alfy. Molecular cell. 2010; 38:265-279. [PubMed: 20417604] 
a

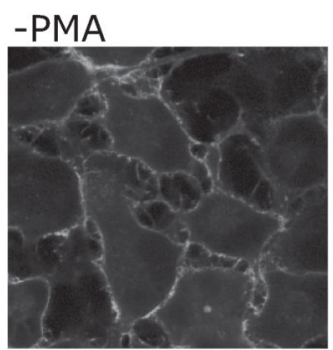

C

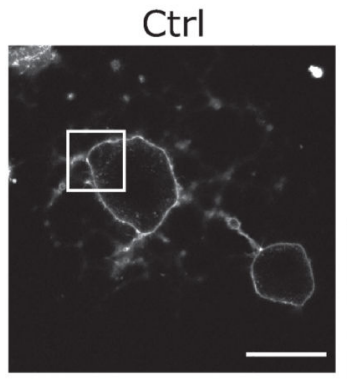

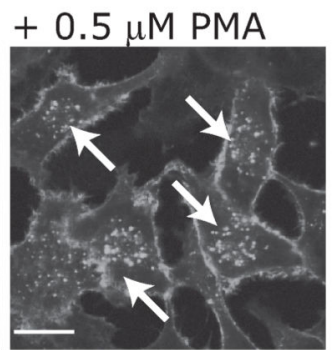

b

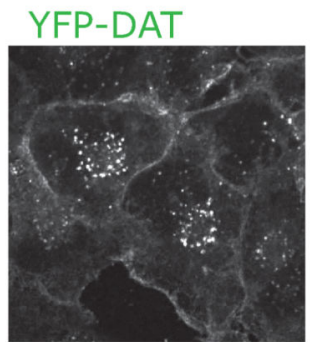

EEA1

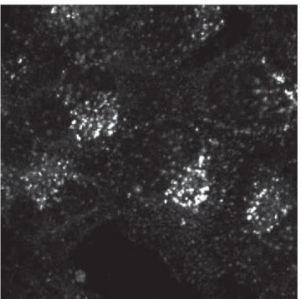

d

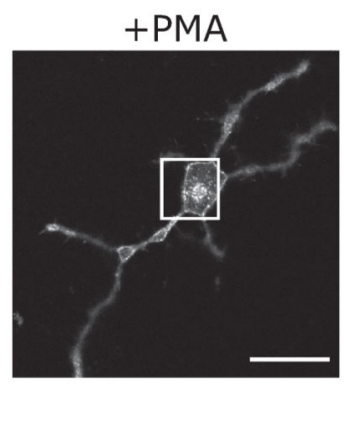

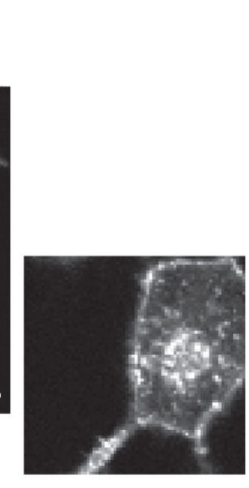
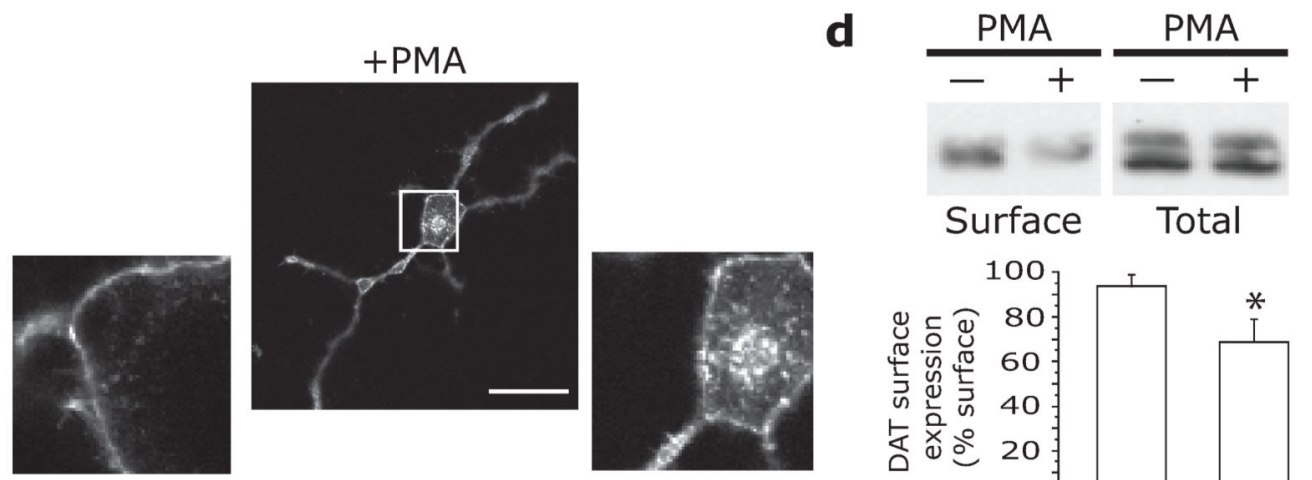

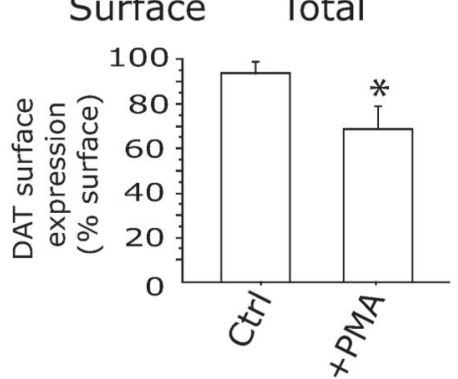

Fig.1. PKC triggers endocytosis of heterologously and endogenously expressed DAT

a,b. EM4-YFP-DAT internalizes (white arrows) into EEA1-positive vesicles after exposure to $0.1 \mu \mathrm{M}$ PMA for $30 \mathrm{~min}$. Cells exposed to PMA were fixed and immunostained for EEA1 as described. Alexa-Fluor 633 labeled secondary antibodies were used to prevent overlap with YFP. Scale bar $=10 \mu \mathrm{m}$. c. Endogenous DAT internalizes in response to PMA in primary dopaminergic neurons. Midbrain cultures were treated with vehicle $(\mathrm{Ctrl}, \mathrm{n}=32)$ or $1 \mu$ M PMA (+PMA, $\mathrm{n}=35$ ) for $30 \mathrm{~min}$, fixed and immunostained for DAT (intracellular epitope, Chemicon), and Alexa-Fluor 568 secondary. PMA led to significant internalization of DAT ('Internalization Index,' as described in Methods, Ctrl: 0.076 \pm 0.013 ; vs. PMA: $0.309 \pm 0.052$ ). (One-way ANOVA; $p<0.001$ ). Scale bar $=20 \mu \mathrm{m}$. d. Internalization of endogenous DAT in striatal slice preparations $(n=6)$. Slices were treated with $10 \mu M$ PMA or Ctrl for $1 \mathrm{hr}$, then cell surface biotinylated to determine DAT surface levels. 'DAT expression levels (\% Surface)' indicates the relative amount of DAT at the cell surface, and was calculated as the ratio between the integrated densities of the surface levels of DAT after treatment (corrected for total levels of DAT) to the surface levels of DAT before treatment (corrected for total levels of DAT). Bars represent Mean + St. Dev. PMA treatment significantly decreased DAT surface levels $(p=0.0330)$. Complete blots can be found in Figure S9. 
a

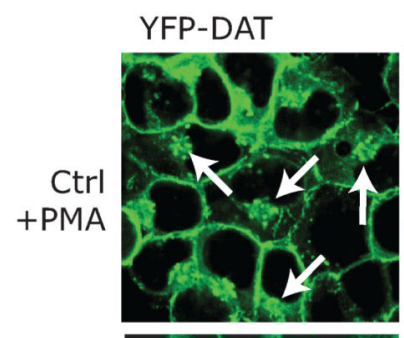

EEA1
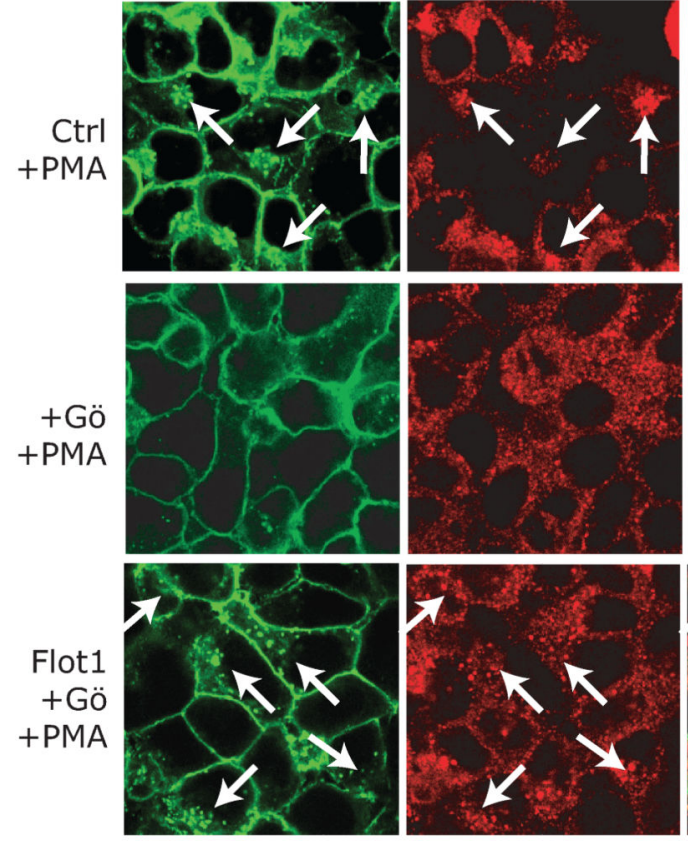

C

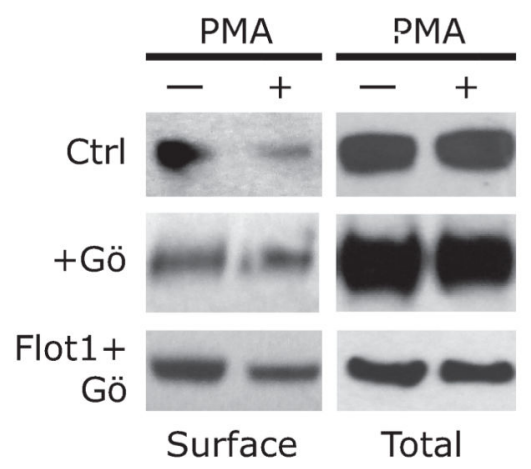

b

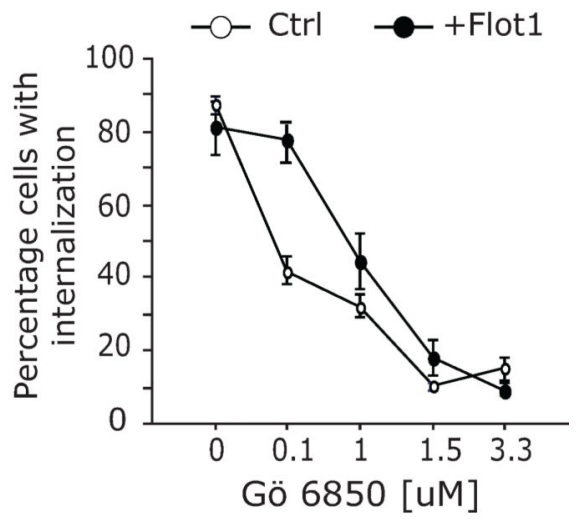

Flot1-RFP
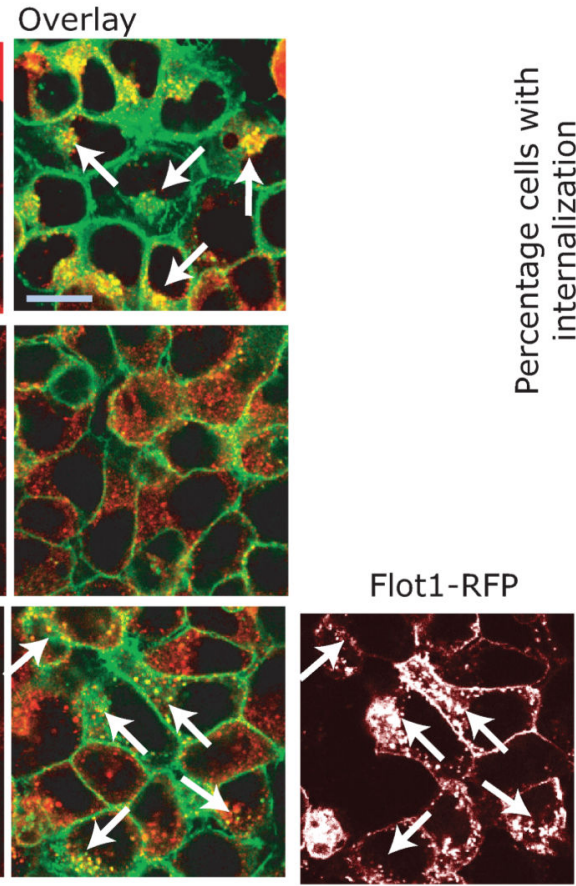

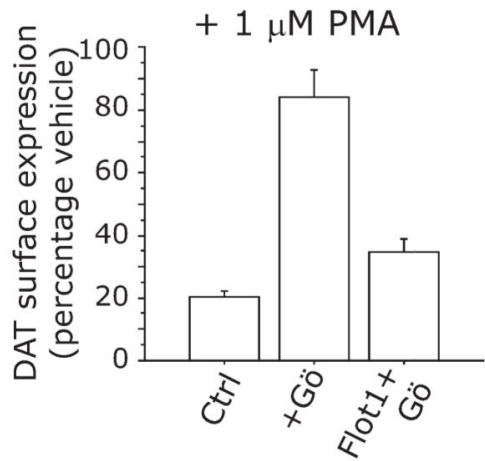

Fig.2. Flot1 overexpression attenuates Gö6850-mediated inhibition of PKC-triggered endocytosis Flot1 overexpression in EM4-YFP-DAT cells attenuated Gö6850-mediated inhibition of PKC-regulated endocytosis. All experiments were performed in this stable cell line unless noted otherwise. a,b. Image based analysis. a. '\% Cell with Internalization' was calculated as cells considered positive for internalization (YFP-DAT co-localized to EEA1-positive endosomes) after $1 \mu \mathrm{M}$ PMA for $30 \mathrm{~min}$. PKC-triggered internalization of DAT was significantly inhibited by Gö6850 in a dose dependent manner (open circles; $\mathrm{p}<0.0001$ ). Flot1 overexpression significantly diminished the Gö6850-mediated inhibition (closed circles, $p=0.0078$ ). For each dose, 150 to 200 cells were analyzed. Scale bar $=10 \mu \mathrm{m}$. Complete statistics can be found in Supporting Materials. b. Flot1 overexpression in the presence of inhibitor (Flot1+ Gö6850) blunted the effect of inhibitor alone, permitting internalization (white arrows). Scale bar $=10 \mu \mathrm{m}$. c. Cell surface biotinylation measuring YFP-DAT surface availability. "DAT surface expression (\% vehicle)" is calculated as 
described (Fig.1d). Values are normalized to no PMA. Data are plotted as Mean + SEM ( $n=$ 5). Ctrl and Gö6850 cells were transfected with mRFP alone as transfection control. Complete blots can be found in Figure. S10. 
a

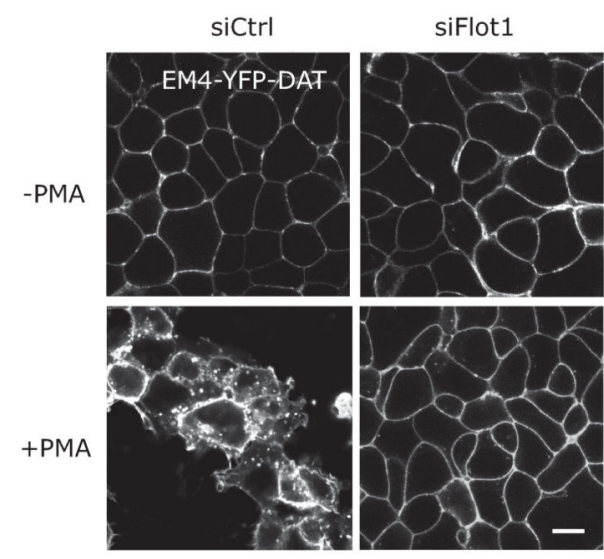

b

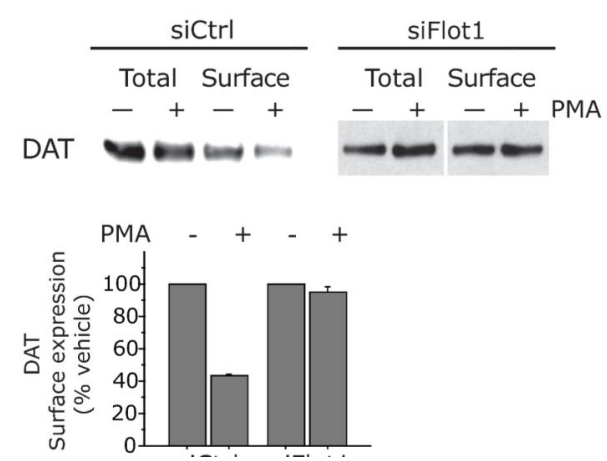

C

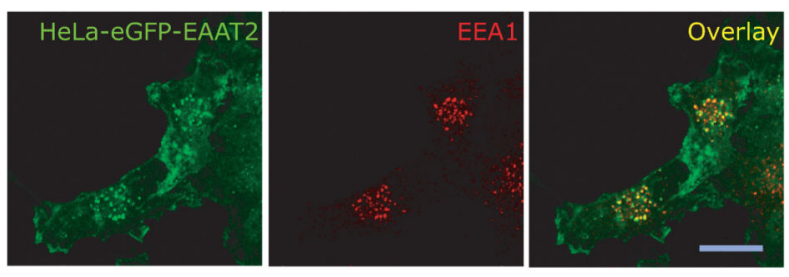

d

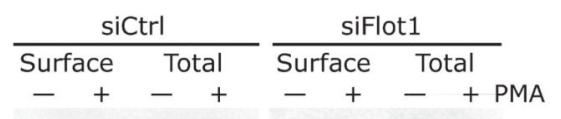

EAAT2

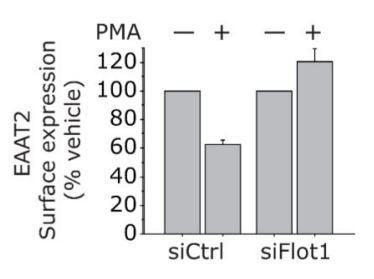

e
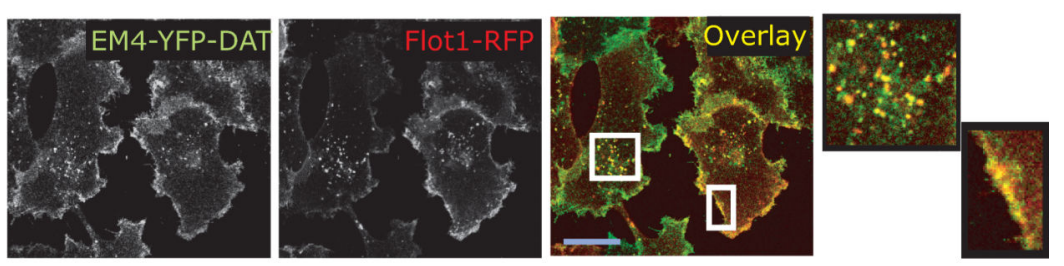

Fig.3. Flot1 is required for the PKC-triggered endocytosis of EM4-YFP-DAT and Hela-eGFPEAAT2

a-b. Flot1 is required for PKC-triggered endocytosis of DAT. EM4-YFP-DAT cells transfected with siRNA against Flot1 (siFlot1) failed to internalize DAT despite exposure to $1 \mu \mathrm{M}$ PMA for $30 \mathrm{~min}$. a. Confocal microscopy $(\mathrm{n}=4)$. b. Cell surface biotinylation $(\mathrm{n}=4)$. Data are plotted as described (Fig.1d). PMA treatment did not lead to a significant decrease of cell surface DAT after Flot1 depletion ( $p=0.226)$. $\mathbf{c}-\mathbf{d}$. Flot1 is required for PKCtriggered endocytosis of EAAT2. c. GFP-EAAT2 internalized to EAA1-positive structures after $0.5 \mu \mathrm{M}$ PMA for $30 \mathrm{~min}$. $(\mathrm{n}=3)$. d. Cell surface biotinylation $(\mathrm{n}=3)$. PMA treatment did not lead to a significant decrease of cell surface EAAT2 after Flot1 depletion ( $\mathrm{p}=$ 0.104). Cells were treated with $0.5 \mu \mathrm{M}$ PMA for $30 \mathrm{~min}$. Complete statistics can be found in Supporting Materials. e. Confocal microscopy revealed that DAT (green) internalized into Flot1-positive vesicles (red) after exposure to $1 \mu \mathrm{M}$ PMA for $30 \mathrm{~min}$ (overlay in yellow).

Scale bars $=10 \mu \mathrm{m}$. Complete blots can be found in Figure S11. 
a

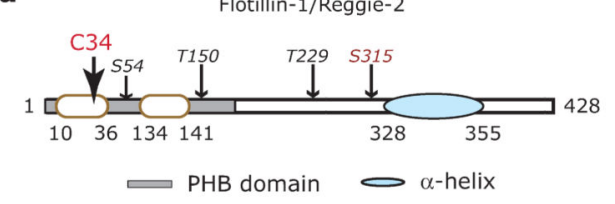

b

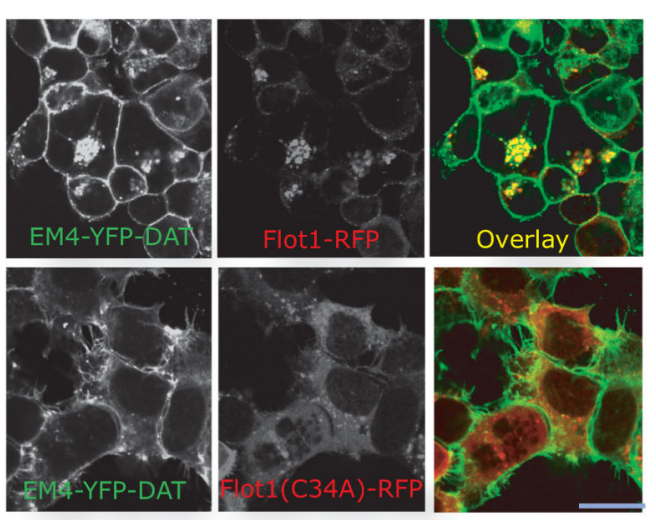

C

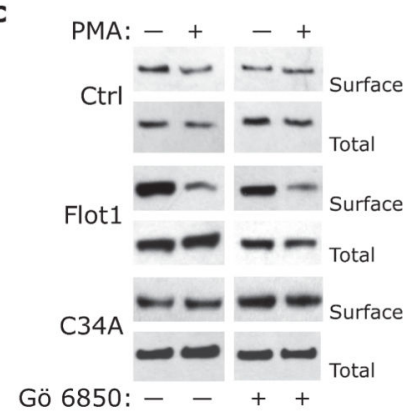

d

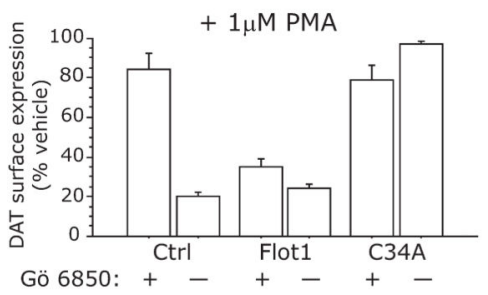

e

f
IP: anti-RFP, IB: anti-Flot1
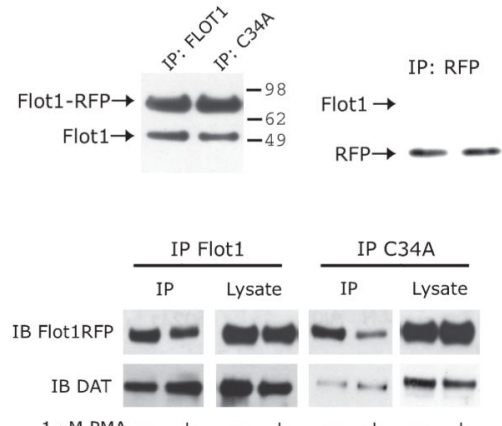

$1 \mu \mathrm{MPMA}-+-+-+-+$

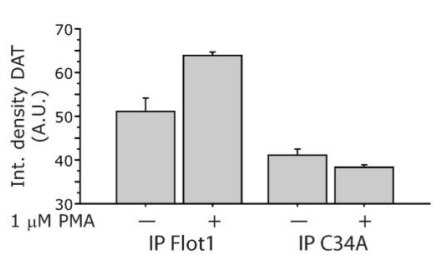

Fig.4. Flot1 must be palmitoylated on residue Cys34

a. Schematic representation of Flot1. The conserved cysteine Cys34 is found within the first hydrophobic domain in the PHB homology domain. Four predicted PKC-phosphorylation sites are also noted. b. Overexpression of Flot1(C34A) in EM4-YFP-DAT inhibited PKCtriggered internalization of DAT ( $n=6$ experiments). Cells transiently transfected with Flot1-mRFP, Flot1(C34A)-mRFP, or mRFP alone (Ctrl), were exposed to $1 \mu \mathrm{M}$ PMA for 30 min. Representative images shown. Scale bar $=10 \mu \mathrm{m}$. c. Cell surface biotinylation of DAT after PMA treatment in the presence or absence of Gö6850. C34A inhibited PMA-triggered internalization (left panels, -Gö6850), and also failed to diminish Gö6850 inhibition (right panels, +Gö6850). d. Quantification of DAT surface expression of c., calculated as described (Fig.1d) $(n=3)$. e. Palmitoylation is not required for multimerization of Flot1. EM4-YFP-DAT cells transiently transfected with mRFP, mRFP-Flot1 or mRFP-

Flot1(C34A). Flot1 fusion proteins were immunoprecipitated with an antibody against mRFP, and probed for endogenous Flot1 (left panel). Flot1(C34A)-mRFP still co-IPed with endogenous Flot1 $(n=3)$. mRFP alone did not co-IP with Flot1 (right panel). $\mathbf{f}$.

Palmitoylation is required for proper complex formation of Flot1 and DAT. Flot1(C34A) pulled-down significantly less DAT than non-mutagenized Flot1. $1 \mu \mathrm{M}$ PMA for 30 min significantly increased the amount of DAT that co-IPs with WT Flot1 $(\mathrm{p}=0.0148)$. This effect was also abrogated by the C34A mutation $(p=0.1022)(n=4)$. Complete statistics can be found in Supporting Materials. Complete blots can be found in Figure S12. 
a

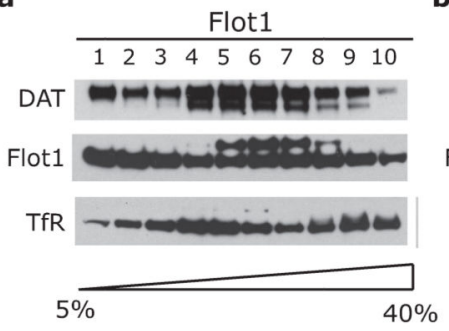

b

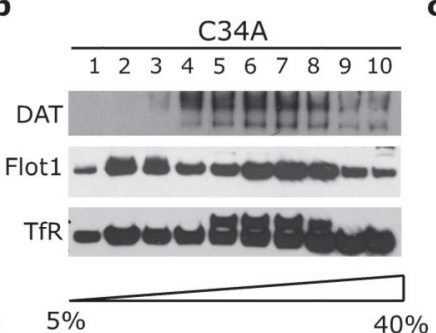
$40 \%$

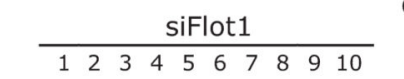
DAT
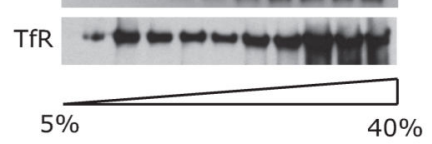

$5 \%$

e
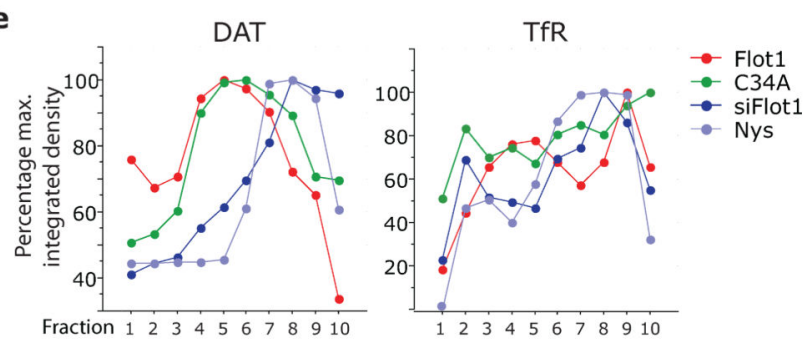

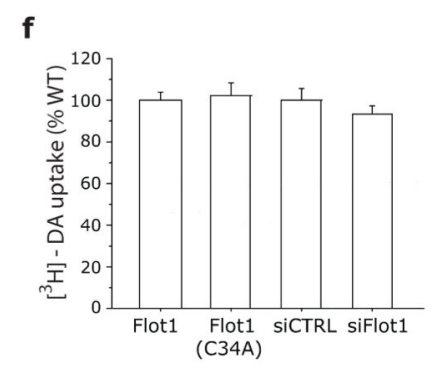

d

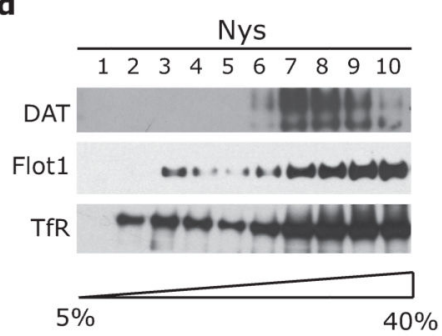

Fig.5. Flot1 is required for the membrane raft localization of DAT but not for transport of DA Sucrose gradients revealed that Flot1 was required to maintain DAT in membrane rafts. EM4-YFP-DAT cells were lysed with $1 \% \mathrm{w} / \mathrm{v}$ Brij58 and fractionated across a discontinuous sucrose gradient. Ten equivolume fractions were collected from top (1) to bottom (10) of the gradient. Four different conditions were run: a. Control conditions (Flot1); b. Flot1(C34A) transfection; c. siFlot1 transfection; and d. nystatin $(25 \mu \mathrm{g} / \mathrm{mL})$ (Nys). $(n=3) . e$. A graphic representation of integrated densities across fractions for each condition is shown for DAT and TfR. f. The transport of DA by DAT does not require the presence of Flot1. EM4-YFP-DAT cells were transfected as indicated, exposed to vehicle, then DA uptake assays were performed as described in Materials. Data is represented as ' $\left[{ }^{3} \mathrm{H}\right]$-DA uptake (\%WT)'. Neither the palmitoylation-deficient mutant nor depletion of Flot1 significantly altered DA uptake $(n=4, p=0.5770)$. Please also refer to Table 1 . Complete blots can be found in Figure S13. 
a
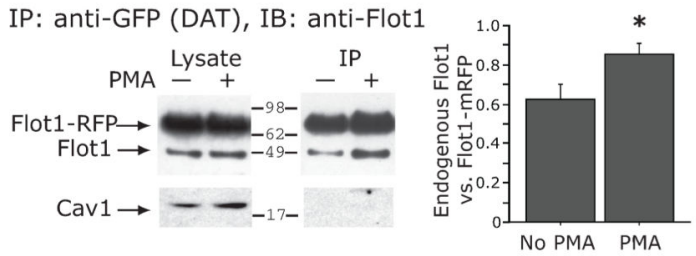

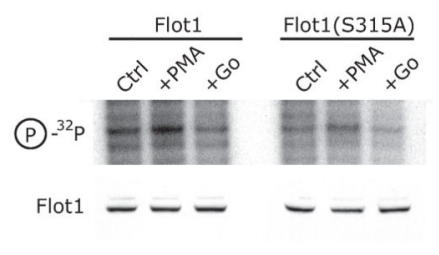

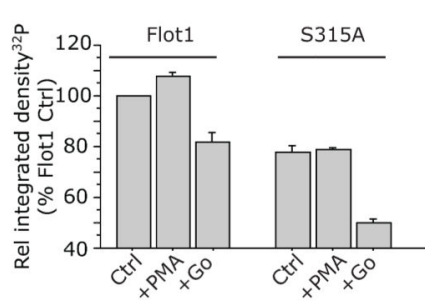

d

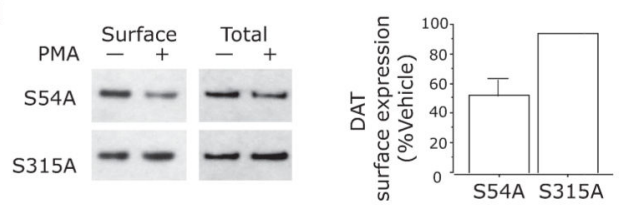

e

H.sapiens 284 RKPAEAERYKLERLAEAEKSQLIMQAEAEAASVRMRGEAEAFAIGARARAEAEG 337 M.Musculus 284 RKPAEAERYRLERLAEAEKAQLIMQAEAEAESVRMRGEAEAFAIGARARAEAEQ 337 R.norvegicus 284 RKPAEAERYRLERLAEAEKAQLIMOAEAEAESVRMRGEAEAFAVGARARAEAEQ 337 C.auratus 284 MKPADAERYRLERLAEAERLQLIMEAEAEAESIKMRGEAEAFAVEARGRAEAEQ 337 D.rerio 284 RKPAEAERYRIEKLAEAERLQLIMEAEAEAESIRMKGEAEAFALEAKGRAEAEQ 337 D.melanogaster 284 RRPAEAEKFRMEKLAEANKQRVVMEAEAEAESIRIRGEAEAFAIAAKAKAEAEQ 337 D.discoideum 487 K--AETDNIALEQKGKA----IIAEAQAKLESAQK--QAQALLITAEAQKKVQE 532 (Vacuolin B)

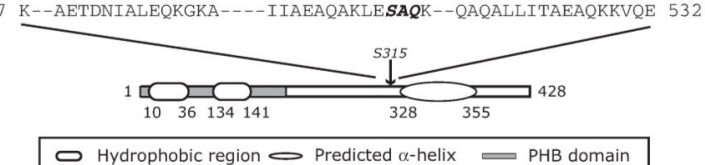

Fig.6. PKC-triggered internalization of DAT requires phosphorylation on Flot1Ser315 a. PMA $(1 \mu \mathrm{M}, 30 \mathrm{~min})$ promotes association of Flot1 with DAT. Bars represent the Mean ratio between integrated densities of endogenous Flot1 to Flot1-mRFP+SEM (n=4). PMA significantly increased mRFP-Flot1 and Flot1 to co-IP with DAT (ANOVA, $p=0.0421$ ). Cav1 did not immunoprecipitate with this complex. b. Metabolic labeling reveals that Flot1 was phosphorylated in response to PMA. EM4-YFP-DAT were treated with vehicle (Ctrl), $1 \mu \mathrm{M}$ PMA + vehicle (+PMA) or $1 \mu \mathrm{M}$ PMA + $100 \mathrm{nM}$ Gö6850 pre-incubation $(+\mathrm{Go})(\mathrm{n}=2)$. Bars represent the integrated density of ${ }^{32} \mathrm{P}$ incorporation normalized to Flot1 levels+SEM. ANOVA reveals +PMA led to a significant increase in ${ }^{32} \mathrm{P}$ incorporation $(\mathrm{p}=0.0474)$ that was inhibited by Gö6850 ( $\mathrm{p}=0.0017)$. S315A mutation inhibited this PMA-triggered increase $(\mathrm{p}=0.1610)$. Overall ${ }^{32} \mathrm{P}$ incorporation was also significantly less in S315A mutant ( $\mathrm{p}<0.001)$. c.-e.. PMA $(1 \mu \mathrm{M}, 30 \mathrm{~min})$-triggered internalization of DAT requires Flot1Ser315. Flot1(S315A) overexpression inhibited DAT internalization while Flot1(S54A) did not. c. DAT is internalized in non-transfected (white arrows) and Flot1(S54A) transfected cells (yellow arrows), but not in Flot1(S315) transfected cells (yellow arrow). '\% Cells with internalization' was determined as described (Fig.2a) (200 cells/condition) (ANOVA, $\mathrm{p}<0.01$ ). Scale bar $=5 \mu \mathrm{m}$. d. Surface protein biotinylation and quantification of DAT $(n=3)$ (ANOVA, $p<0.01)$. e. Alignment of region surrounding Flot1Ser315. Ser315 is largely conserved across species (*, bold). Complete statistics can be found in Supporting Materials. Complete blots can be found in Figure S14. 
a

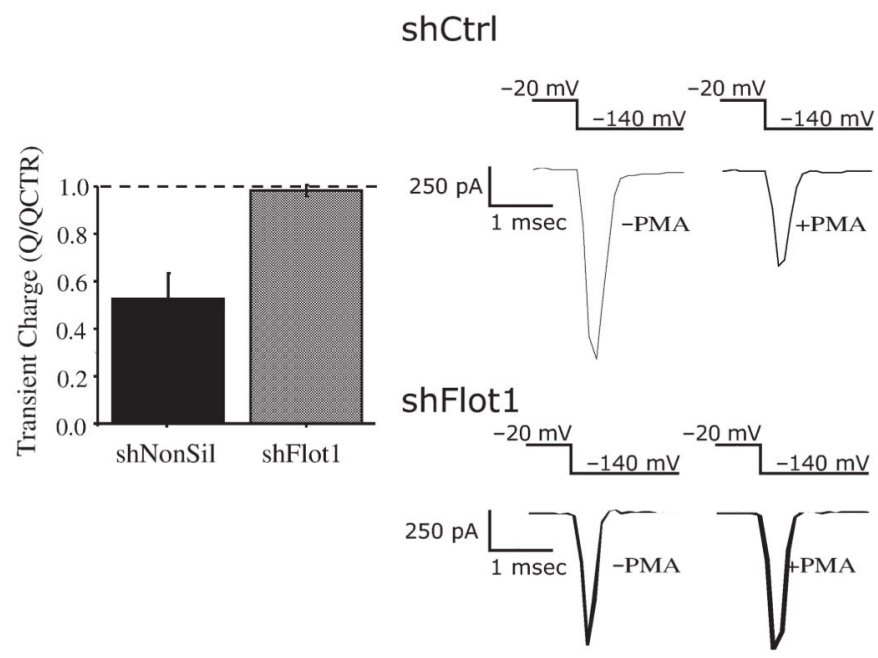

b

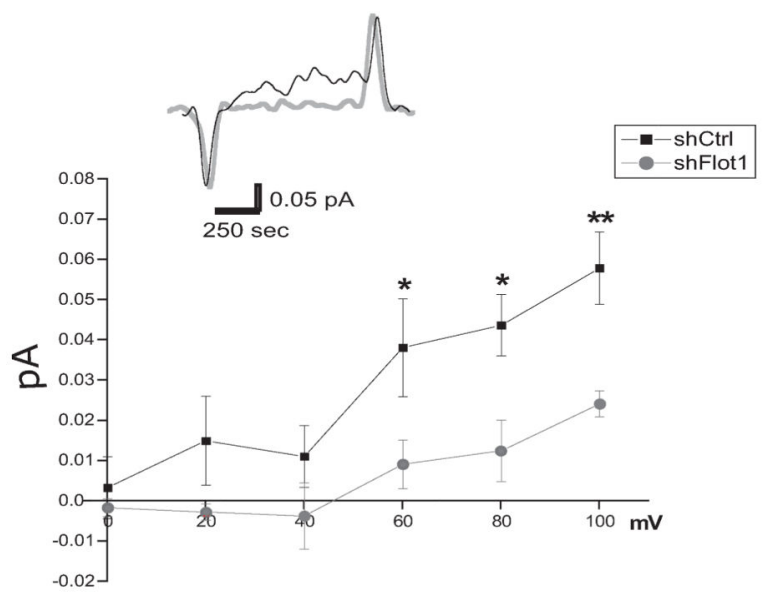

Fig.7. Flot1 is required for PKC-triggered internalization and AMPH-induced reverse transport of DA in primary DAergic neurons

a. Representative DAT-mediated transient currents recorded from DA neurons transduced with lentiviri carrying shCtrl or shFlot 1 following a voltage jump to $-140 \mathrm{mV}$ from a holding potential of $-20 \mathrm{mV}$. The integral of the DAT-mediated transient current is proportional to the number of transporters on the cell surface2. Transient currents were obtained first with vehicle, then in the presence of PMA. Bars represent the mean ratios of PMA transient currents to pre-PMA responses in the same cell. PMA did not reduce DATmediated transient currents in shFlot1 neurons, suggesting that Flot1 is necessary for PMAtriggered DAT trafficking. b. Loss of Flot1 leads to an inhibition of AMPH-mediated DA efflux. Neurons were loaded via the patch pipette with $2 \mathrm{mM}$ DA and $30 \mathrm{mM} \mathrm{Na}^{+}$. Amperometric current-voltage relationship was obtained by stepping the voltage in $20 \mathrm{mV}$ intervals from $0 \mathrm{mV}$ to $+100 \mathrm{mV}$ from a holding potential of $-60 \mathrm{mV}$. DAT-mediated DA efflux is defined as the current recorded in the presence of AMPH, minus the current recorded after the addition of cocaine to the bath with AMPH still present from shFlot1 (red, $\mathrm{n}=4$ ) and shCtrl (black, $\mathrm{n}=4$ ). Values are reported as mean \pm SEM (* indicates $\mathrm{p}<0.05$ ). shFlot1 significantly reduced DA efflux as compared to shCtrl at voltages greater than +40 $\mathrm{mV}(* \mathrm{p}<0.05, * * \mathrm{p}<0.01$, One-way ANOVA followed by Bonferroni posthoc tests). 


\section{Table I}

Tyramine uptake competition assay.

\begin{tabular}{|rcc|}
\hline & $\begin{array}{c}\text { Tyramine } \\
\mathbf{K}_{\mathbf{M}}(\boldsymbol{\mu M})\end{array}$ & $\begin{array}{c}\mathbf{V}_{\mathbf{M A X}} \\
(\boldsymbol{\%})\end{array}$ \\
\hline Flot1 WT & $2.30[1.25-4.22]$ & 100 \\
Flot1(C34A) & $2.17[1.19-3.19]$ & $95 \pm 3.2$ \\
\hline siCTRL & $2.73[1.99-3.74]$ & 100 \\
siFlot1 & $2.69[1.67-4.32]$ & $91 \pm 24$ \\
\hline Ctrl & $6.04[5.27-6.92]$ & 100 \\
Nys & $5.86[5.00-6.76]$ & $103 \pm 6.6$ \\
\hline & &
\end{tabular}

The Km values were calculated from the observed IC50 value found by non-linear regression analysis of $\left[{ }^{3} \mathrm{H}\right]$ tyramine uptake competition assays. Data obtained from EM4 cells transiently expressing YFP-DAT. Data are means + SEM of 2-5 experiments performed in duplicate. The SEM interval for each Km value is indicated in brackets and was calculated from the pKI + SEM The calculated Vmax for $\left[{ }^{3} \mathrm{H}\right]$ tyramine uptake was normalized to the respective control values after normalization for cell density. 